\title{
Gestão de projetos em empresas no Brasil: abordagem "tamanho único"?
}

\section{Project management in companies in Brazil: a "one size fits all" approach?}

\section{Luiz José Marques Junior ${ }^{1}$ Guilherme Ary Plonski ${ }^{1}$}

\begin{abstract}
Resumo: A pequena taxa de sucesso dos projetos num cenário de importância crescente para realização das estratégias das empresas levanta questionamentos sobre a efetividade das abordagens de gestão de projetos. A revisão da bibliografia sobre o tema sugere a existência de uma abordagem predominante de gestão de projetos que utiliza técnicas e ferramentas de planejamento e controle com o propósito de racionalizar e normatizar a gestão de projetos. Em contraponto a essa abordagem tradicional predominante, surgiu a abordagem adaptativa. Nela, a gestão de cada projeto é adaptada às suas especificidades. Neste contexto, este artigo apresenta o resultado de um estudo exploratório preliminar sobre abordagens de gestão de projetos em seis empresas que atuam no Brasil. Os dados empíricos coletados permitem concluir que há predominância de abordagens focadas numa visão racional e normativa da gestão de projetos. Porém, os mesmos dados coletados também indicam a presença de práticas de abordagens adaptativas na gestão de projetos considerados estratégicos, sugerindo que não há abordagem "tamanho único" na gestão de projetos dessas empresas.
\end{abstract}

Palavras-chave: Gestão de projetos. Abordagens gerenciais.

\begin{abstract}
The low success rate of projects in a scenario where they are increasingly important for the companies' strategies raises questions about the effectiveness of project management approaches. A literature review on this topic suggests that there is a predominant approach to project management, which makes use of planning and control tools and techniques to encourage a rational and normative perspective to project management. As a counterpoint to this predominant traditional approach, the adaptive approach has emerged arguing that the management of each project should be adapted to its specificities. In this context, this paper presents the result of a preliminary exploratory study about project management approaches on six companies in Brazil. The empirical data collected allow concluding that the approaches focused on a rational and normative view of project management are predominant. On the other hand, the data also indicated the presence of adaptive approach practices in the management of strategic projects suggesting that there is no "one size fits all" approach to manage projects in the companies studied.
\end{abstract}

Keywords: Project management. Management approaches.

\section{Introdução}

No ambiente competitivo dos negócios, os projetos têm papel importante na gestão estratégica das organizações. Os projetos são os vetores das mudanças, da implementação das estratégias e das inovações que trazem vantagens competitivas para as empresas. Cleland (1994) já dizia que nenhuma organização pode escapar do ritmo incansável das mudanças geradas por tecnologias de produto e processo que mudam rapidamente, por ciclos de vida de serviços e produtos cada vez mais curtos e por tecnologias da informação em constante evolução.
Apesar da importância cada vez maior dos projetos nas organizações, a maioria dos projetos não cumpre suas metas. Entregar projetos que atendam às metas de prazo, custo e especificações planejadas e que também atendam aos objetivos de negócio que o justificaram é ainda um desafio a ser superado nas empresas. Pesquisas indicam que somente $28 \%$ dos projetos de tecnologia da informação têm sucesso, que projetos de construção e reforma têm atrasos médios de $103 \%$ e que somente um em quatro produtos desenvolvidos se torna um sucesso comercial

\footnotetext{
${ }^{1}$ Departamento de Engenharia de Produção, Escola Politécnica da Universidade de São Paulo - USP,

E-mails: luiz.marques@alumni.duke.edu; plonski2@usp.br
} 
(CHAOS REPORT, 1995; MARQUES JUNIOR., 2000; SHENHAR; DVIR, 2007).

Neste cenário de baixas taxas de sucesso nos projetos, é natural que a abordagem predominante de gestão de projetos seja questionada. Na revisão da literatura, identificou-se que esta abordagem predominante, também chamada de abordagem tradicional, tem como foco o desenvolvimento de técnicas e ferramentas de planejamento e controle que racionalizam e normatizam a gestão dos projetos. No entanto, argumenta-se que o uso efetivo de tais técnicas e ferramentas pressupõem um cenário estável e previsível e que tais condições não se verificam mais no atual ambiente de negócios, caracterizado pela instabilidade e imprevisibilidade, em que os projetos são influenciados pelas dinâmicas do ambiente, da tecnologia e dos mercados (WILLIAMS, 2005; MAYLOR, 2001).

A contrapartida da abordagem tradicional é a abordagem adaptativa. $\mathrm{O}$ argumento a favor desta abordagem é que os projetos devem ser reconhecidos como diferentes entre si e que a gestão de cada projeto deve ser adaptada para contemplar essas diferenças em relação aos seus objetivos, tarefas e ambiente. A premissa é que a adoção de uma abordagem contingencial aumenta a chance de sucesso dos projetos nas organizações.

É evidente que as duas abordagens têm pontos a favor e contra. A abordagem tradicional, racional e normativa, pode trazer padronização e simplificação. Por outro lado, sua aplicação a qualquer tipo de projeto é questionável, dadas as diferentes complexidades e incertezas de cada projeto no cenário atual de negócios. A abordagem adaptativa ou contingencial pode ser mais efetiva num cenário instável e imprevisível como o atual, pois a estratégia de gestão de cada projeto é adaptada às suas especificidades. Por outro lado, o grande número de projetos nos portfólios das empresas pode tornar inviável utilizar uma solução para gestão de cada projeto. Além disso, a falta de padronização pode dificultar melhorias nos processos de gestão de projetos.

Portanto, considerou-se a premissa de que existe uma abordagem predominante de gestão de projetos que utiliza técnicas e ferramentas de planejamento e controle com propósito de racionalizar e normatizar a gestão de projetos. Este trabalho procurou verificar se esta premissa se confirma em seis empresas que atuam no Brasil e gerenciam projetos complexos e incertos.

A estruturação do trabalho ficou dividida em introdução, fundamentos teóricos, metodologia, resultados e conclusões. Na revisão bibliográfica que representa o fundamento teórico deste trabalho foi feita inicialmente uma discussão sobre a importância e o desempenho dos projetos dentro do ambiente organizacional. A seguir são apresentados os aspectos que caracterizam a abordagem tradicional de gestão de projetos. As abordagens adaptativas ou contingenciais são discutidas a seguir. Ao final do tópico é apresentado o modelo teórico utilizado para a pesquisa objeto deste trabalho.

A metodologia utilizada foi uma pesquisa exploratória com empresas que atuam no Brasil e tenham projetos complexos e incertos em seus portfólios. Inicialmente é feita uma justificativa para o tipo de estudo adotado, seguido pelos critérios adotados para a seleção das unidades de análise e as limitações do estudo.

No item de resultados, é apresentada a tabela dos resultados obtidos nas entrevistas e feita uma análise comparativa dos resultados obtidos entre empresas utilizando o modelo teórico definido na revisão da bibliografia. Finalmente, na conclusão, é feita a relação dos resultados obtidos com os objetivos propostos no estudo e são feitas recomendações.

\section{Fundamentos teóricos}

Com a alta demanda por crescimento e inovação, os projetos passaram a ter importância cada vez maior nas organizações. De acordo com Shenhar e Dvir (2007), a globalização dos mercados força as empresas a responderem demandas locais e a competirem por baixo custo no mundo todo. $\mathrm{Da}$ mesma forma, as tecnologias da informação e da Internet provocaram uma revolução de mudanças nas empresas que continuam em curso. Os projetos são iniciativas únicas, como lançar novos produtos, novas organizações ou novos empreendimentos, ou ainda, melhorar produtos existentes e investir na infraestrutura da empresa. Para Cleland (1994), projetos são os elementos executores de mudanças nas organizações que permitem às organizações sobreviver e crescer.

Apesar da importância cada vez maior dos projetos nas organizações, a maioria dos projetos não cumpre suas metas iniciais de prazo, custo, qualidade e negócios. Marques Junior (2000) aponta atrasos médios de $103 \%$ numa pesquisa com 398 projetos de construção no setor público. Pesquisa da KPMG's International 2002-2003 mostra que $61 \%$ dos projetos de TI fracassaram em uma amostra de 176 empresas públicas e privadas do Canadá. O Chaos Report (1995) aponta que $31,1 \%$ dos projetos de tecnologia da informação foram cancelados antes de terminarem. Outro número do mesmo relatório mostra que 52,7\% dos projetos tiveram aumento médio de $189 \%$ nos custos. Shenhar e Dvir (2007) pesquisaram 600 projetos nos setores privado, governamental e terceiro setor em vários países e constataram que $85 \%$ dos 
projetos não cumpriram o prazo e orçamento originais, com atraso médio de $70 \%$ e aumento médio de $60 \%$ no orçamento.

As causas apontadas para o não cumprimento das metas dos projetos são diversas. Marques Junior (2000) aponta os fatores ligados ao planejamento como a causa mais frequente dos problemas em projetos de construção na área pública. Hartman e Ashrafi (2002) definem causas críticas: falta de definição do que seja sucesso, falta de apoio da alta administração, falta de métricas para controle e falta de alinhamento dos interessados com critérios de sucesso. Na pesquisa KPMG's International 2002-2003, as causas são o planejamento deficiente, a falta de alinhamento com o negócio e a falta de envolvimento da alta administração. Black (1996), em pesquisa com 70 engenheiros, aponta a falta de definição no início do projeto como a causa principal de atrasos e estouros de orçamento. Em projetos de web design, Fichter (2003) relaciona as principais causas de problemas: falta de envolvimento do usuário, falta de apoio dos executivos da empresa, falta de definição clara dos requisitos e planejamento deficiente.

Historicamente, o combate às causas de insucessos dos projetos tem foco no desenvolvimento e aperfeiçoamento de ferramentas e técnicas de planejamento e controle (MORRIS, 1994; PACKENDORFF, 1995; THOMITOCLEOS; WEARNE, 2000; THOMAS, 2000; KLOPPENBORG; OPFER, 2002; LEYBOURNE, 2007). Este foco levou a uma visão predominantemente positivista, tecnocentrada e racional da gestão de projetos, com predomínio de teorias normativas. A premissa assumida nestas teorias é que basta aplicar corretamente um conjunto padronizado de procedimentos, técnicas e ferramentas para se ter sucesso nos projetos.

Segundo Packendorff (1995), esta premissa pode ser observada em casos relatados na literatura. A maioria deles é de exemplos de sucesso, que mostram os benefícios de se usar técnicas e ferramentas de planejamento e controle. Os exemplos de fracassos são imputados ao uso incorreto ou ao não uso das técnicas e ferramentas de planejamento e controle. Ainda segundo Packendorff (1995), a predominância da abordagem tradicional teve impulso com o surgimento dos softwares no início da década de 1980 e o seu crescente desenvolvimento, com preços acessíveis e sofisticação, que permitiram aos praticantes da gestão de projetos aplicar as técnicas de planejamento numa ampla escala de projetos.

$\mathrm{Na}$ abordagem tradicional de gestão de projetos, os processos de planejamento, organização, direcionamento e controle da equipe são definidos para atender a metas e objetivos estabelecidos (CLELAND, 1994). A premissa fundamental é que um projeto tem uma tarefa definida e que, portanto, os esforços do gerente de projeto e de sua equipe devem ser dirigidos para o uso eficiente de recursos e técnicas de planejamento e controle (PACKENDORFF, 1995).

$\mathrm{O}$ posicionamento positivista da abordagem tradicional pressupõe que a realidade existe e pode ser observada, permitindo assim definir uma referência de melhores práticas. Segundo Smyth e Morris (2007), em pesquisa feita em artigos publicados no ano de 2005 no International Journal of Project Management, o positivismo tem sido dominante nas pesquisas sobre gestão de projetos. O positivismo busca a generalização para estabelecer princípios ou leis que regem um fenômeno.

Na década de 1990, o surgimento e disseminação dos documentos de orientação de boas práticas, conhecidos como Bodies of Knowledge-BoKs em gestão de projetos, contribuiram para renovar o foco no planejamento e controle dos projetos e para reforçar a visão normativa e prescritiva da gestão de projetos, caracterizada pela premissa implícita de que basta seguir as melhores práticas que os projetos terão sucesso (PACKENDORFF, 1995; WILLIAMS, 2005). Segundo o guia para o Project Management Body of Knowledge - PMBoK (PROJECT..., 2004), o sistema de gestão de projetos é formado por processos associados a nove áreas de conhecimento que o gerente de projetos deve dominar para, em conjunto com aspectos comportamentais (liderança, comunicação interpessoal, gestão de conflitos, etc.), atender às metas do projeto. Os grupos de processos são: iniciação, planejamento, execução, controle e encerramento. As áreas de conhecimento são: gestão da integração, gestão do escopo, gestão do tempo, gestão dos recursos humanos, gestão de custos, gestão das aquisições, gestão da qualidade, gestão do risco, gestão da comunicação. O PMBoK (2004) é normativo e positivista, pois pretende servir de referência de melhores práticas na gestão de projetos.

A abordagem tradicional pode ser observada na descrição que Cleland (1994) sugere para os passos de uma programação de projetos:

- Definir os objetivos, metas e estratégias gerais do projeto.

- Desenvolver a Work Breakdown Structure - WBS.

- Sequenciar os pacotes de trabalho e atividades.

- Estimar os elementos de tempo e custo.

- Revisar a programação máster em face das restrições de tempo do projeto.

- Reconciliar a programação com as restrições de recursos organizacionais.

- Revisar a consistência da programação em relação aos objetivos de custo e de desempenho técnico do projeto.

- Aprovar a programação junto à gerência sênior. 
De maneira similar, Shenhar e Dvir (2007) descrevem a abordagem tradicional ou convencional de gestão de projetos nos seguintes passos: o processo é iniciado com a criação de uma declaração de escopo, que define o trabalho que precisa ser feito, com os principais produtos/serviços a serem entregues. Este escopo é então decomposto em pacotes de trabalho, organizados hierarquicamente numa estrutura analítica de projeto - EAP. Com a EAP é construída a estrutura organizacional do projeto. A partir daí é montada a rede de atividades do projeto, sobre a qual são alocados os recursos necessários, finalizando com a montagem do cronograma do projeto. Outros elementos do plano do projeto são então agregados. O objetivo do controle dos projetos é garantir a realização do projeto dentro do prazo, dentro do orçamento, e de acordo com as especificações.

As críticas à abordagem tradicional de gestão de projetos são concentradas em sua inadequação para gestão de projetos complexos e incertos nas organizações. Pesquisas têm indicado a inconsistência das premissas de racionalidade das abordagens tradicionais: os planos de projeto não são estáveis; os procedimentos de planejamento servem mais para legitimar o projeto do que para guiá-lo; ferramentas sofisticadas de planejamento raramente são usadas; planos precisos nem sempre são as ferramentas gerenciais mais úteis (PACKENDORFF, 1995; KREINER, 1995; MAYLOR, 2001; MILLS et al., 2002; MIDLER, 2002; MURIITHI; CRAWFORD, 2003; JAAFARI, 2003; WILLIAMS, 2005; CICMIL, 2006; HALLGREEN; MAANINEN-OLSSON, 2005; LEYBOURNE, 2007; SHENHAR; DVIR, 2007; HARTMAN, 2008).

Estas críticas desencadearam a busca de novas abordagens para adaptar a gestão às condições de incerteza e complexidade associadas às metas, atividades e ao ambiente no qual os projetos estão inseridos. Um projeto está submetido a influências desconhecidas que podem surgir da ambiguidade e da complexidade do projeto. Ambiguidade se refere à falta de consciência da equipe do projeto sobre certos eventos ou seus efeitos. Complexidade se refere à dificuldade de avaliar o efeito de uma ação específica no projeto devido à interação de diferentes ações com parâmetros de eventos (PICH; LOCH; MEYER, 2002).

A ambiguidade e a complexidade dos projetos trazem implicações para a gestão de projetos, por exemplo, a necessidade de ciclos de iterações entre projeto de produto e testes no desenvolvimento de novos produtos. Tais iterações são muito importantes em ambientes que exigem tempos curtos de lançamento do produto no mercado (EISENHARDT; TABRIZI, 1995). Portanto, como os projetos não são fundamentalmente iguais, existe a necessidade de identificar características importantes para uso mais apropriado de técnicas e ferramentas de gestão de projetos (PINTO; SLEVIN, 1988).

Os estudos sobre abordagens adaptativas de gestão de projetos focam as diferenciações e classificações de projetos, as práticas adaptativas aos diferentes tipos de projetos e aos critérios de sucesso (PINTO; SLEVIN, 1988; MORRIS, 1994; PACKENDORFF, 1995; ATKINSON, 1999; PICH; LOCH; MEYER,2002; MILLS et al., 2002; JAAFARI, 2003; WESTERVELD, 2003; SHENHAR; DVIR, 2007; DONK; MOLLOY, 2008).

A abordagem adaptativa sugerida por Shenhar e Dvir (2007) para gestão de projetos nas organizações apresenta um modelo flexível e adaptativo direcionado para o sucesso. Os projetos passam de um conjunto de atividades a serem entregues no prazo e no custo para processos relacionados a negócios que devem entregar resultados de negócio. A intenção do modelo é disciplinar a análise de benefícios e riscos esperados de um projeto e desenvolver um conjunto de regras e comportamentos para cada tipo de projeto. Segundo Shenhar e Dvir (2007), o modelo proposto pode ajudar a atacar a causa raiz dos fracassos dos projetos. $\mathrm{O}$ resultado de pesquisas realizadas por eles em cerca de 600 projetos foi que os executivos e equipes de projetos falham na avaliação inicial das incertezas e complexidades dos projetos e/ou comunicação dos resultados dessas análises e falham também na adaptação dos estilos gerenciais à situação específica de cada projeto.

Shenhar e Dvir (2007) sugerem classificar cada projeto conforme seu posicionamento em quatro dimensões: inovação, tecnologia, complexidade e ritmo.

- Inovação: representa a incerteza da meta do projeto e/ou a incerteza no mercado.

- Tecnologia: representa a incerteza tecnológica.

- Complexidade: mede a complexidade do produto, tarefa, e organização.

- Ritmo: representa a urgência do projeto.

Cada uma dessas dimensões é dividida em graduações. A dimensão inovação é dividida em derivativo, plataforma e ruptura. A dimensão da incerteza da tecnologia a ser empregada no projeto é dividida em baixa, média, alta e superalta tecnologia. A dimensão complexidade é dividida em montagem, sistema e grande sistema. Por fim, a dimensão ritmo é dividida em regular, competitivo, crítico e blitz.

O modelo teórico adotado na pesquisa está baseado fundamentalmente no trabalho de Shenhar e Dvir (2007), que consolida cerca de 15 anos de pesquisa sobre gestão adaptativa de projetos. O modelo teórico utilizado neste trabalho é apresentado na Tabela 1 e considera tópicos de gestão de projetos divididos conforme o ciclo de vida de um projeto: fase de definição, fase de planejamento, fase de execução e 
Tabela 1. Modelo teórico do estudo. Fonte: Shenhar and Dvir, 2007.

Atividade Detalhes

Fase de definição

Documento formal de início

Identificação do tipo de projeto

Critérios de sucesso e fracasso

Planejamento

Impacto do tipo de

projeto na gestão

Plano de gestão de requisitos

Plano de criação do produto

\section{WBS}

Comunicação

Organização

Plano de processos

Programação

Orçamentação

Gestão dos riscos

Plano de integração

Aquisições

Qualidade

Recursos humanos

Plano de monitoramento

Fase de execução

Requisitos do produto

Construção do produto

\section{Monitoramento}

Replanejamento

Fase de encerramento

Preparação para o consumidor

Comercialização

Fechamento do projeto
Identificação do mercado/consumidor; Necessidade do consumidor; Definir o objetivo de negócio esperando que seja atingido quando o projeto acabar; Descrição do produto; Requisitos do produto; Escopo;

Duração aproximada; Orçamento aproximado; Gerente e equipe do projeto

Categorizar o projeto baseado em metas estratégicas ou operacionais, usuários internos ou externos, inovação, tecnologia, complexidade, ritmo e outras dimensões relevantes.

Definir as expectativas gerenciais em cinco ou mais dimensões de sucesso, e definir o que pode dar errado.

Decidir como cada categoria de projeto afetará a organização, processos, planos, atividades, e equipe.

Estimar as incertezas dos requisitos iniciais; Coleta de dados de mercado; Quantidade de protótipos de mercado; Momento do congelamento de requisitos

Especificações técnicas iniciais; Design inicial do produto; Quantidade de ciclos de design; Momento de congelamento do design; Plano de testes do produto

Quebrar o escopo em pacotes de trabalho e atividades

Estrutura dos reportes e agenda de reuniões; Meios e tecnologias de comunicação - local, global

Estrutura da equipe de projeto; Matriz de responsabilidades

Principais fases, portões e marcos

Rede detalhada e momento das atividades

Custo detalhado do projeto conforme o WBS

Identificação dos riscos e mitigação baseada no tipo de projeto

Momento e duração das atividades de integração

Plano de subcontratações e fornecimentos

Plano de gestão da qualidade

Desenvolvimento da equipe e treinamento

Atividades de motivação da equipe

Revisões do projeto, planos e decisões necessárias nos portões

Refinamento dos requisitos do produto;

Congelamento dos requisitos de produto

Design do produto; Construção e teste do protótipo; Ciclos adicionais de design (redesenho, reconstrução e reteste); Congelamento das especificações e do design do produto; Construção do produto; Teste do produto

Posição das atividades, dos prazos e do orçamento realizados

Atualizar os planos e fazer mudanças

Documentação; Materiais e meios de treinamento; Simulação de uso do produto Plano de introdução do produto; Assimilação do produto;

Distribuição do produto

Relatório resumo do projeto; Relatório de lições aprendidas; Planejamento da nova geração 
fase de encerramento. Dentro destas fases do ciclo de vida são destacados os tópicos relacionados à abordagem adaptativa em itálico negrito.

\section{Metodologia}

Neste estudo foi utilizada a pesquisa exploratória, uma vez que o objetivo da pesquisa é verificar a premissa de que a abordagem tradicional de gestão de projetos é predominante. Para tanto foram analisadas as práticas de gestão de projetos de seis empresas que atuam no Brasil para identificar a aderência das práticas à abordagem tradicional e adaptativa, conforme definida no modelo teórico apresentado na Tabela 1. Segundo Gil (2002), as pesquisas exploratórias são desenvolvidas com o objetivo de apresentar uma visão geral acerca de um determinado fato, sem a pretensão de generalizá-lo, nem tampouco esgotar o assunto.

Outra característica da pesquisa exploratória é constituir-se, muitas vezes, de uma primeira etapa de investigação mais ampla em que o tema escolhido é amplo, tornando-se necessário delimitá-lo para que seu produto final passe a ser um problema mais claro, no qual, posteriormente, poderão ser realizados estudos mediante a utilização de procedimentos mais sistematizados.

Neste caso, o estudo apresentado constituiu-se de um estudo preliminar sobre abordagens tradicional e adaptativa de gestão de projetos, tendo como fator motivador a falta de conhecimento sobre a aderência das práticas de gestão de projetos das empresas em relação às duas abordagens de gestão de projetos identificadas na literatura.

Para realizar o levantamento dos dados necessários, foi realizado um estudo de campo que, de acordo com Gil (2002), é recomendado para analisar a interação dos componentes de um grupo especifico. O grupo específico é constituído por empresas de porte médio ou grande que atuam no Brasil e têm projetos complexos e incertos em seus portfólios. Foi formulado um roteiro para as entrevistas conduzidas junto a pessoas dos quadros gerenciais e de supervisão de seis empresas que atenderam os critérios de seleção da pesquisa. A Tabela 2 apresenta a lista dos setores econômicos de cada uma das empresas.
Para selecionar as empresas estudadas, foram utilizados os seguintes critérios:

- Empresas que estejam gerenciando ou tenham gerenciado recentemente projetos com pelo menos duas das seguintes características:

- Escopo amplo (abrangência, quantidade de interfaces);

- Uso de tecnologia pouco conhecida;

- Produto gerado pelo projeto seja inovador para o consumidor (interno ou externo); e

- Tempo disponível para implantação seja crítico.

\section{- Facilidade de acesso do pesquisador}

Na pesquisa foram utilizados os seguintes instrumentos de coleta de dados: entrevista semiestruturada, observação direta e pesquisa em arquivos. As entrevistas foram concedidas por profissionais das empresas selecionadas que tiveram envolvimento na gestão de projetos complexos e incertos.

Para os procedimentos de campo de uma pesquisa, Yin (2005) recomenda que o pesquisador atente para os seguintes pontos quanto aos procedimentos: obter acesso a organizações ou entrevistados-chave; possuir recursos suficientes enquanto estiver no campo; desenvolver um procedimento para pedir ajuda e orientação; estabelecer uma agenda clara das atividades de coleta de dados; preparar-se para mudanças inesperadas.

A pesquisa que foi feita identificou as empresas potenciais e as pessoas de contato para a realização do estudo exploratório. Os recursos para a coleta dos dados foram um bloco de notas e um gravador de voz para registro das entrevistas. As ajudas e orientações vieram de conversas com outros professores e colegas. A coleta de dados foi feita nos meses de outubro, novembro e dezembro de 2008. As entrevistas tiveram duração de 45 minutos até uma hora e meia. Dois terços das entrevistas foram gravados e transcritos ipsis verbis para posterior análise dos dados.

Além das entrevistas, nesta pesquisa, foi utilizada a pesquisa em arquivos para obtenção de dados secundários. Esta pesquisa foi feita em web sites das empresas, em publicações sobre as empresas em revistas não acadêmicas e em documentos internos

Tabela 2. Setores econômicos das empresas pesquisadas. Fonte: Elaborado pelos autores.

\begin{tabular}{ccc}
\hline Empresa & Setor & Quantidade de entrevistas \\
\hline 1 & Bens de consumo & 2 \\
2 & Siderurgia & 1 \\
3 & Financeiro & 3 \\
4 & Mineração & 1 \\
5 & Bens de consumo & 2 \\
6 & Agrícola & 3 \\
\hline
\end{tabular}


das empresas que foram disponibilizados para o pesquisador. Numa etapa anterior às entrevistas foi realizada uma análise de conteúdo das fontes secundárias disponíveis, buscando informações que ajudassem na elaboração dos casos. Por exemplo, foram analisados os relatórios de relacionamento com investidores para tentar identificar projetos com as características de complexidade e incerteza descritas anteriormente.

Essa pesquisa tem limitantes decorrentes do método de pesquisa utilizado. Primeiro, os resultados e conclusões dessa pesquisa mostram apenas a aderência ou não aderência das práticas de gestão de projetos das empresas da amostra à abordagem tradicional e adaptativa, não sendo possível generalizar os resultados para todas as empresas que atuam no Brasil, ou mesmo para outras empresas dos setores econômicos estudados. Segundo limitante é a escolha arbitrária do referencial teórico da pesquisa, baseado na abordagem de gestão adaptativa de Shenhar e Dvir (2007). Evidentemente, outros modelos de gestão adaptativa poderiam ser usados. Demais limitantes dessa pesquisa foram: a escolha da amostra de empresas, com critérios pré-estabelecidos, mas dentro da conveniência do pesquisador; e a entrevista única em duas das seis empresas estudadas.

No entanto, apesar dos limitantes da pesquisa, os resultados obtidos podem constituir-se em fortes indícios para elaboração de futuras pesquisas ou aprofundamentos deste estudo.

\section{Resultados}

Os resultados obtidos nas entrevistas nas seis empresas estão apresentados nas Tabelas 3 e $4 . \mathrm{Na}$ análise dos resultados, foram observadas diferenças e similaridades nas práticas de gestão de projetos, sob a ótica do referencial teórico baseado em Shenhar e Dvir (2007). Na definição do projeto, pode-se verificar que predomina a formalidade para início dos projetos pelos documentos iniciais do projeto. Porém, a diferenciação dos projetos é limitada a uma classificação entre estratégico e não estratégico. Dentro da classificação de projeto estratégico, não foi observada uma diferenciação para posicionamento destes projetos quanto à complexidade e à incerteza envolvida. Na empresa 4, apesar dos projetos serem classificados em várias dimensões, a diferenciação de complexidade e incerteza entre os projetos não é clara. Ainda na definição do projeto, pode-se verificar que os projetos são avaliados por critérios multidimensionais. Além do atendimento do prazo, custo e especificações, os projetos são avaliados em critérios operacionais, de segurança, meio ambiente e critérios de impacto no negócio.

No planejamento, foram constatadas estratégias predominantes entre as empresas estudadas. No quesito de impacto do tipo de projeto na gestão, observou-se que os projetos estratégicos seguem uma metodologia formal de gestão, baseada no PMBoK (PROJECT..., 2004), com apoio de escritórios de projeto. Os projetos não estratégicos não têm uma metodologia padrão e não são monitorados pelos escritórios de projeto. No entanto, entre os projetos estratégicos não há diferenciação de aplicação da metodologia. O roteiro é o mesmo para todos os projetos estratégicos. A predominância do uso de estruturas de quebra do escopo (WBS - Work Breakdown Structure), de planos de comunicação e organização, de programações e orçamentos detalhados reforça a constatação da existência de uma metodologia comum a todos os projetos estratégicos. Vale ressaltar que a empresa 3 e a empresa 6 não tem uma metodologia padrão para condução de seus projetos estratégicos.

Ainda no planejamento, nos quesitos de gestão dos requisitos e de plano de construção do produto, observou-se que as empresas utilizam estratégias de minimização das incertezas nos projetos estratégicos. Predomina entre as empresas a utilização de pilotos de escala reduzida, pesquisas de mercado e grupos foco para entender melhor os requisitos iniciais do projeto estratégico. Predominaram também análises de incertezas técnicas na construção dos produtos, serviços ou resultados do projeto. No entanto, não foi percebida a existência de análises formais para definir a intensidade de uso destas estratégias entre os projetos estratégicos. No quesito de gestão de riscos, observou-se a predominância de análises de risco formais entre as empresas estudadas em seus projetos estratégicos. Lembrando novamente que, por não haver diferenciação formal entre os projetos estratégicos, a análise de riscos não é diretamente influenciada pelas diferentes complexidades e incertezas dos projetos estratégicos.

Na execução dos projetos, percebe-se uma divisão de práticas. Nos quesitos de requisitos do produto e construção do produto, observou-se a divisão entre empresas que levam em conta o contingenciamento necessário para eventuais ajustes e correções nos produtos, serviços ou resultados dos projetos estratégicos. Nos requisitos do produto, metade das empresas leva em conta tempos de contingenciamento para eventuais mudanças. Na construção do produto, apenas uma empresa atribui contingenciamentos para mais de um ciclo de design e testes para eventuais correções e ajustes. Ainda na execução de projetos, percebeu-se que há preocupação predominante em definir intervalos de controle dos projetos e em ter um controle formal de mudanças. Lembrando novamente que, por não diferenciar seus projetos estratégicos, a intensidade dos contingenciamentos, a frequência de controle e o rigor do controle de mudanças são, a princípio, uniformes para qualquer 
Tabela 3. Resultados das empresas 1, 2 e 3. Fonte: Elaborado pelos autores.

\begin{tabular}{|c|c|c|c|}
\hline Atividade & Empresa 1 & Empresa 2 & Empresa 3 \\
\hline \multicolumn{4}{|l|}{ Fase de definição } \\
\hline $\begin{array}{l}\text { Documento formal de } \\
\text { início }\end{array}$ & Sim & Sim & Não \\
\hline $\begin{array}{l}\text { Identificação do tipo de } \\
\text { projeto }\end{array}$ & Estratégico/Rotina - formal & Estratégico/Rotina - formal & $\begin{array}{c}\text { Estratégico/Rotina - não } \\
\text { formal }\end{array}$ \\
\hline $\begin{array}{l}\text { Critérios de sucesso e } \\
\text { fracasso }\end{array}$ & $\begin{array}{l}\text { Prazo,custo,indica-dores } \\
\text { operacionais, financeiros }\end{array}$ & $\begin{array}{l}\text { Prazo, custos, operacionais, } \\
\text { segurança e negócio }\end{array}$ & $\begin{array}{c}\text { Prazo, custo, cliente, } \\
\text { negócio }\end{array}$ \\
\hline \multicolumn{4}{|l|}{ Fase de planejamento } \\
\hline $\begin{array}{l}\text { Impacto do tipo de projeto } \\
\text { na gestão do projeto }\end{array}$ & $\begin{array}{c}\text { Projetos estratégicos têm } \\
\text { metodologia PMBoK } \\
\text { formal e única }\end{array}$ & $\begin{array}{l}\text { Projetos estratégicos têm } \\
\text { metodologia PMBoK } \\
\text { formal e única }\end{array}$ & Não é feita formalmente \\
\hline $\begin{array}{l}\text { Plano de gestão de } \\
\text { requisitos }\end{array}$ & $\begin{array}{c}\text { Protótipos de mercado e } \\
\text { pesquisas }\end{array}$ & Protótipos de mercado & $\begin{array}{l}\text { Grupos foco, pesquisas e } \\
\text { pilotos }\end{array}$ \\
\hline $\begin{array}{l}\text { Plano de criação do } \\
\text { produto }\end{array}$ & $\begin{array}{c}\text { Faz avaliação incerteza } \\
\text { técnica }\end{array}$ & $\begin{array}{c}\text { Contingência para ciclos de } \\
\text { design e testes }\end{array}$ & $\begin{array}{l}\text { Não dimensiona tempos de } \\
\text { contingenciamento }\end{array}$ \\
\hline WBS & Sim & Sim & Não \\
\hline Comunicação & Sim & Não & Não \\
\hline Organização & Sim & Sim & Não \\
\hline $\begin{array}{l}\text { Plano de processos do } \\
\text { projeto }\end{array}$ & Sim & Sim & $\operatorname{Sim}$ \\
\hline Programação & Sim & Sim & Não \\
\hline Orçamentação & Sim & Não & Não \\
\hline Gestão dos riscos & $\begin{array}{l}\text { Tipo de projeto não } \\
\text { influencia na contingência }\end{array}$ & $\begin{array}{l}\text { Tipo de projeto não } \\
\text { influencia na contingência }\end{array}$ & $\begin{array}{l}\text { Tipo de projeto não } \\
\text { influencia na contingência }\end{array}$ \\
\hline Plano de integração & Sim & Sim & Não \\
\hline Aquisições & Sim & Sim & Não \\
\hline Qualidade & Sim & Sim & Sim \\
\hline Recursos humanos & Não & Não & Não \\
\hline $\begin{array}{l}\text { Plano de monitoramento do } \\
\text { projeto }\end{array}$ & Sim & Sim & Não \\
\hline \multicolumn{4}{|l|}{ Execução } \\
\hline Requisitos do produto & $\begin{array}{c}\text { Considera } \\
\text { contingenciamento }\end{array}$ & $\begin{array}{c}\text { Não leva em conta } \\
\text { contingenciamento - } \\
\text { anualizado }\end{array}$ & $\begin{array}{l}\text { Não leva em conta } \\
\text { contingenciamento }\end{array}$ \\
\hline Construção do produto & $\begin{array}{c}\text { Não considera ciclos de } \\
\text { design e testes }\end{array}$ & $\begin{array}{c}\text { Não considera ciclos de } \\
\text { design e testes }\end{array}$ & $\begin{array}{c}\text { Não considera ciclos de } \\
\text { design e testes }\end{array}$ \\
\hline Monitoramento do projeto & Quinzenal & $\begin{array}{c}\text { Mensal - Metas } \\
\text { Anualizadas }\end{array}$ & $\begin{array}{c}\text { Sem intervalos pré- } \\
\text { definidos }\end{array}$ \\
\hline Replanejamento do projeto & $\begin{array}{l}\text { Controle formal de } \\
\text { mudanças }\end{array}$ & $\begin{array}{l}\text { Controle formal de } \\
\text { mudanças }\end{array}$ & $\begin{array}{c}\text { Não há padronização de } \\
\text { controle }\end{array}$ \\
\hline \multicolumn{4}{|l|}{ Fase de encerramento } \\
\hline $\begin{array}{l}\text { Preparação para o } \\
\text { consumidor }\end{array}$ & $\begin{array}{c}\text { Sim, transferência para } \\
\text { usuário }\end{array}$ & $\begin{array}{c}\text { Sim, transferência para } \\
\text { operações }\end{array}$ & $\begin{array}{l}\text { Sim, transferência para } \\
\text { área requisitante }\end{array}$ \\
\hline Comercialização & Não aplicável & Não aplicável & Não aplicável \\
\hline Fechamento do projeto & Relatório final & Não há formalização & Não há formalização \\
\hline
\end{tabular}


Tabela 4. Resultados das empresas 3, 4 e 5. Fonte: Elaborado pelos autores.

\begin{tabular}{|c|c|c|c|}
\hline Atividade & Empresa 4 & Empresa 5 & Empresa 6 \\
\hline \multicolumn{4}{|l|}{ Fase de início } \\
\hline $\begin{array}{l}\text { Documento formal de } \\
\text { início }\end{array}$ & Sim & Sim & Não \\
\hline $\begin{array}{l}\text { Identificação do tipo de } \\
\text { projeto }\end{array}$ & $\begin{array}{c}\text { Classificação em várias } \\
\text { dimensões }\end{array}$ & $\begin{array}{l}\text { Investimento ou } \\
\text { Sustentação }\end{array}$ & Programa ou Projeto \\
\hline $\begin{array}{l}\text { Critérios de sucesso e } \\
\text { fracasso }\end{array}$ & $\begin{array}{l}\text { Prazo, custo, } \\
\text { operacionais,segu-rança, } \\
\text { meio ambiente e negócio }\end{array}$ & $\begin{array}{l}\text { Prazo, custo, operacionais, } \\
\text { segurança e negócio }\end{array}$ & Prazo, custo e cliente \\
\hline \multicolumn{4}{|l|}{ Fase de planejamento } \\
\hline $\begin{array}{l}\text { Impacto do tipo de projeto } \\
\text { na gestão do projeto }\end{array}$ & $\begin{array}{c}\text { Projetos estratégicos } \\
\text { seguem metodologia Front } \\
\text { End Loading -FEL }\end{array}$ & $\begin{array}{c}\text { Projetos de Investimento } \\
\text { seguem metodologia } \\
\text { PMBoK }\end{array}$ & Não é feita formalmente \\
\hline $\begin{array}{l}\text { Plano de gestão de } \\
\text { requisitos }\end{array}$ & Prevê ciclos de iteração & Falta formalização & Não há avaliação formal \\
\hline $\begin{array}{l}\text { Plano de criação do } \\
\text { produto }\end{array}$ & $\begin{array}{l}\text { Incerteza técnica é } \\
\text { considerada }\end{array}$ & $\begin{array}{c}\text { Tempos de } \\
\text { contingenciamento são } \\
\text { dimensionados }\end{array}$ & $\begin{array}{c}\text { Não faz avaliação formal } \\
\text { da incerteza técnica }\end{array}$ \\
\hline WBS & Sim & Sim & Não \\
\hline Comunicação & Sim & Sim & Não \\
\hline Organização & Sim & Sim & Não \\
\hline $\begin{array}{l}\text { Plano de processos do } \\
\text { projeto }\end{array}$ & Sim & Sim & Não \\
\hline Programação & Sim & Sim & Não \\
\hline Orçamentação & Sim & Sim & Não \\
\hline Gestão dos riscos & $\begin{array}{l}\text { Tipo de projeto não } \\
\text { influencia na contingência }\end{array}$ & $\begin{array}{l}\text { Tipo de projeto não } \\
\text { influencia na contingência }\end{array}$ & $\begin{array}{l}\text { Tipo de projeto não } \\
\text { influencia na contingência }\end{array}$ \\
\hline Plano de integração & Sim & Sim & Não \\
\hline Aquisições & Sim & Sim & Não \\
\hline Qualidade & Sim & Sim & Não \\
\hline Recursos humanos & Não & Não & Não \\
\hline $\begin{array}{l}\text { Plano de monitoramento do } \\
\text { projeto }\end{array}$ & Sim & Sim & Não \\
\hline \multicolumn{4}{|l|}{ Fase de execução } \\
\hline Requisitos do produto & $\begin{array}{c}\text { Considera } \\
\text { contingenciamento }\end{array}$ & $\begin{array}{c}\text { Considera } \\
\text { contingenciamento }\end{array}$ & $\begin{array}{l}\text { Não leva em conta } \\
\text { contingenciamento }\end{array}$ \\
\hline Construção do produto & $\begin{array}{c}\text { Considera ciclos de design } \\
\text { e testes }\end{array}$ & $\begin{array}{l}\text { Não considera ciclos de } \\
\text { design e testes }\end{array}$ & $\begin{array}{l}\text { Não considera ciclos de } \\
\text { design e testes }\end{array}$ \\
\hline Monitoramento do projeto & Mensal & Mensal & Sem frequência definida \\
\hline Replanejamento do projeto & $\begin{array}{l}\text { Controle formal de } \\
\text { mudanças }\end{array}$ & $\begin{array}{l}\text { Controle formal de } \\
\text { mudanças }\end{array}$ & $\begin{array}{c}\text { Não há controle de } \\
\text { mudanças }\end{array}$ \\
\hline \multicolumn{4}{|l|}{ Fase de Encerramento } \\
\hline $\begin{array}{l}\text { Preparação para o } \\
\text { consumidor }\end{array}$ & $\begin{array}{c}\text { Sim, transferência para } \\
\text { operações }\end{array}$ & $\begin{array}{c}\text { Sim, transferência para } \\
\text { operações }\end{array}$ & $\begin{array}{l}\text { Sim, transferência de } \\
\text { tecnologia }\end{array}$ \\
\hline Comercialização & Não aplicável & Não aplicável & Não aplicável \\
\hline Fechamento do projeto & Relatório final & Relatório final & Relatório final \\
\hline
\end{tabular}


projeto estratégico, estando sujeitas à variação por decisão direta do gestor.

No encerramento dos projetos, as empresas estudadas têm práticas similares. Como a maioria segue uma metodologia baseada no PMBoK (PROJECT..., 2004), observou-se a presença de relatórios finais de fechamento e avaliação dos projetos.

\section{Conclusões e recomendações}

A análise dos resultados do estudo preliminar com as seis empresas traz dois indícios em relação à verificação da premissa de predominância da abordagem tradicional de gestão de projetos: um deles confirmatório e o outro contraditório.

O indício confirmatório foi a predominância da abordagem tradicional, baseada no racionalismo e na normatização, de acordo com as visões da abordagem tradicional de gestão de projetos sugeridas por Cleland (1994), Williams (2005) e Shenhar e Dvir (2007). A evidência mais contundente da predominância da abordagem tradicional nas práticas de gestão de projetos nas seis empresas foi a utilização do referencial do PMBoK (PROJECT..., 2004) na construção das metodologias de gestão dos projetos nas empresas estudadas.

O indício contraditório foi a verificação da utilização de práticas contidas na abordagem adaptativa, apesar da predominância da abordagem tradicional. Uma destas práticas é definição de estratégias diferenciadas de gestão entre projetos estratégicos e não estratégicos. Os projetos estratégicos têm metodologias próprias e são acompanhados pelos escritórios de projeto (Project Management Office - PMO). Outra prática é a utilização de critérios de sucesso multidimensionais para os projetos estratégicos. Isto mostra que as empresas se preocupam em definir o sucesso de seus projetos de maneira mais ampla, além do tradicional triângulo de ferro: prazo, custo e qualidade. Outra prática observada é a utilização de estratégias diferenciadas de gestão de requisitos e construção do produto nos projetos estratégicos. Isto mostra que as empresas, de alguma maneira, consideram as incertezas na definição de requisitos de projetos com maior carga de inovação.

No entanto os indícios de utilização da abordagem adaptativa são limitados. Entre os projetos considerados estratégicos pelas empresas estudadas, não há diferenciação formal quanto à complexidade $\mathrm{e}$ incerteza. Por não haver diferenciação, a abordagem de gestão de todos os projetos estratégicos se baseia numa metodologia uniforme, única para todos eles. Shenhar (2001) havia chegado à conclusão similar em estudo com empresas ao constatar que, implicitamente, as empresas utilizam estratégias diferentes para diferentes projetos, mas não há uma identificação do tipo de projeto e a consequente adaptação do estilo gerencial antes de iniciar o projeto.

Uma implicação desta constatação é que as empresas perdem a oportunidade de estabelecer uma gestão de projetos mais efetiva em seus projetos estratégicos. Ao criar diferenciações entre os projetos estratégicos, as empresas poderiam entender melhor a complexidade e incerteza envolvida em cada projeto e assim identificar riscos importantes dos projetos logo no início do ciclo de vida. Ao entender melhor os riscos iniciais, executivos e gerentes de projeto teriam melhores condições de definir estratégias de implantação que minimizassem ou evitassem riscos.

Outra implicação para utilização limitada da abordagem adaptativa em projetos estratégicos pode ser a falta de um referencial teórico consistente. As práticas tradicionais têm o referencial do PMBoK (PROJECT..., 2004), predominantemente utilizado pelas empresas estudadas, mas as práticas adaptativas ainda não têm um pilar referencial predominante. Neste sentido, Shenhar e Dvir (2007) apresentaram um modelo de gestão adaptativa para projetos, mas ainda não há evidências empíricas sobre a efetividade da sua utilização.

\section{Referências}

ATKINSON, R. Project management: cost, time and quality, two best guesses and a phenomenon, it is time to accept other success criteria. International Journal of Project Management, v. 17, n. 6, 1999.

BLACK, K. Causes of project failure: a survey of professional engineers. PM Network, p. 21-24, 1996.

CHAOS REPORT. The Standish Group Report, 1995. Disponível em http://www.scs.carleton.ca/ beau/PM/ Standish-Report.html.

CICMIL, S. Understanding project management practice through interpretative and critical research perspectives. Project Management Journal, v. 37, n. 2, 2006.

CLELAND, d. i. Project management: strategic design and implementation. 2. ed. McGraw-Hill, 1994.

DONK, D. P. VAN; MOLLOY, E. From organising as projects to projects as organizations. International Journal of Project Management, v. 26, p. 129-137, 2008.

EISENHARDT, K. M.; TABRIZI, B. N. Accelerating adaptive processes: product innovation in the global computer industry. Administrative Science Quarterly, v. 40, p. 84-110, 1995.

FICHTER, D. Why web projects fail. Online, 2003. Disponível em: <http://www.allbusiness.com/sciencetechnology/standards-measurements/10606809-1.html>. Acesso em: 05 maio 2008.

GIL, A. C. Como elaborar projetos de pesquisa. São Paulo: Atlas, 2002.

HALLGREEN, M.; MAANINEN-OLSSON, E. Deviations, ambiguity and uncertainty in a project-intensive organization. Project Management Journal, v. 36, n. 3, p. 17-26, 2005. 
HARTMAN, F. Preparing the mind for dynamic management. International Journal of Project Management, v. 26, p. 258-267, 2008.

HARTMAN, F.; ASHRAFI, R. Project Management in the information technology and information systems industry. Project Management Journal, v. 33, n. 3, p. $5-15,2002$.

JAAFARI, A. Project Management in the age of complexity and change. Project Management Journal, v. 34, n. 4, p. 47-57, 2003.

KLOPPENBORG, T. J.; OPFER, W. A. Forty years of project management research: trends, interpretations, and predictions. In: SLEVIN, D. P.; CLELAND, D. I.; PINTO, J. K. The frontiers of project management research. Project Management Institute, 2002. p. 3-30.

KPMG KPMG's International 2002-2003. Programme management survey. U.K.: KPMG, 2002.

KREINER, K. In search of relevance: project management in drifting environments. Scandinavian Journal of Managament, v. 11, n. 4, p. 335-346, 1995.

LEYBOURNE, S. A. The changing bias of project management research: a consideration of the literatures and an application of extant theory. Project Management Journal, v. 38, n. 1, p. 62-73, 2007.

MARQUES JUNIOR, L. J. Uma contribuição para melhoria do planejamento de empreendimentos de construção em organizações públicas. 2000. Dissertação (Mestrado)-Escola Politécnica da Universidade de São Paulo, São Paulo, 2000.

MAYLOR, H. Beyond the gantt chart: project management moving on. European Management Journal, v. 19, p. 92-100, 2001.

MIDLER, C. Project management for intensive, innovationbased strategies: new challenges for the $21^{\text {st }}$ century. In: SLEVIN, D. P.; CLELAND, D. I.; PINTO, J. K. The frontiers of project management research. Project Management Institute, 2002.

MILLS, B. et al. Managing technological innovation projects: the quest for a universal language. In: SLEVIN, D. P.; CLELAND, D. I.; PINTO, J. K. The Frontiers of Project Management Research. Project Management Institute, 2002.

MORRIS, P. W. G. The management of projects. Thomas Telford, 1994.

MURIITHI, N.; CRAWFORD, L. Approaches to project management in Africa: implications for international development projects. International Journal of Project Management, v. 21, p. 309-319, 2003.

PACKENDORFF, J. Inquiring into the temporary organization: new directions for Project management research. Scandinavian Journal of Management, v. 11, n. 4, 1995.

PICH, M. T.; LOCH, C.; MEYER, A. E. On uncertainty, ambiguity, and complexity in project management. Management Science, v. 48, n.8, p. 1008-1023, 2002.

PINTO, J. K.; SLEVIN, D. P. Project Success: definitions and measurement techniques. Project Management Journal, v. 19, n. 1, p. 67-72, 1988.

PMBoK: a guide to the project management body of knowledge. Project Management Institute, 2004.

SHENHAR, A. J. One size does not fit all projects: exploring classical contingency domains. Management Science, v. 47, n. 3, p. 394-414, 2001.

SHENHAR, A. J.; DVIR, D. Reinventing project management: the diamond approach to successful growth and innovation. Boston: Harvard Business School Press, 2007.

SMYTH, H. J.; MORRIS, P. W. G. A epistemological evaluation of research into projects and their management: methodological issues. International Journal of Project Management, v. 25, p. 423-436, 2007.

The frontiers of project management research. Editado por Dennis P. Slevin; David I. Cleland e Jeffrey K. Pinto. Project Management Institute, 2002.

THOMAS, J. L. Making sense of project management: contingency and sensemaking in transitory organizations. Thesis (Doctor of Philosophy)-Faculty of Graduate Studies and Research, University of Alberta, Edmonton, Alberta, Canada, 2000.

THOMITOCLEOS, G.; WEARNE, S. H. Project management topic coverage in journals. Journal of Project Management, v. 18, n. 1, p. 7-11, 2000.

WESTERVELD, E. The Project Excellence Model: linking success criteria and critical success factors. International Journal of Project Management, v. 21, p. 411-418, 2003.

WILLIAMS, T. Assessing and moving on from the dominant Project management discourse in the light of Project overruns. IEEE Transactions on Engineering Management, v. 52, n. 4, p. 497-508, 2005.

YIN, R. K. Estudo de caso: planejamento e métodos. 3. ed. Bookman, 2005 